\title{
Comportamento e desempenho de vacas leiteiras no período de transição de sete dias antes e após o parto
}

\section{Feeding behavior and performance of dairy cows during the transition period seven days before and after calving}

\author{
Mário José Giannasi Scala ${ }^{1 *}$; José Antonio Fregonesi²; Ivone Yurika Mizubuti; \\ Daniel Martin Weary ${ }^{4}$; Marina Andrea Gräfin von Keyserlingk4; Douglas Veira ${ }^{5}$; \\ Andressa Amorim Cestari ${ }^{6}$; Lizanne Steunenberg ${ }^{7}$
}

\begin{abstract}
Resumo
Durante o período de transição as vacas leiteiras passam por vários desafios internos e externos, que podem deixá-las sujeitas a problemas de saúde como acidose do rúmen e pododermatite. O manejo tradicional durante o período de transição recomenda o fornecimento de uma ração com teores médios de concentrado no pré-parto, e outra com teores mais altos de concentrado no pós-parto. Entretanto, existem evidências de que o consumo de rações com alto teor de concentrado após o parto pode levar a irregularidades no consumo destas dietas. Objetivou-se estudar o efeito de manter constante a composição da dieta sobre o comportamento, consumo de alimentos e produção de vacas leiteiras durante o período de transição. Trinta vacas primíparas e multíparas da raça Holandesa foram divididas e alocadas em dois grupos, sendo um deles tratado com ração constante pré-parto (30:70 relação concentrado:volumoso) com maior teor de fibras por sete dias após o parto, seguindo a troca de ração para a dieta tradicional (50:50 relação volumoso:concentrado); e outro grupo, recebendo ração com maior teor de concentrados no pós-parto. O comportamento e o consumo de alimentos foram monitorados com sistema eletrônico de alimentação e data loggers, e a produção de leite através de extração de dados direto do computador da ordenhadeira mecânica. Vacas leiteiras alimentadas com dieta constante durante o período de transição despenderam mais tempo comendo ração no segundo dia pós-parto, 142 minutos/dia, e vacas no tratamento com dieta tradicional 105 minutos/dia. Aquelas manejadas com troca de dieta no pós-parto apresentaram maior consumo no primeiro dia, IMS de $2,2 \mathrm{~kg} / \mathrm{dia} / 100 \mathrm{~kg}$ PV, mas não nos seguintes, já para as vacas que receberam a dieta constante a IMS foi de $1,7 \mathrm{~kg} /$ dia/100 kg PV. Não foram observadas diferenças significativas na produção de leite em relação às duas dietas oferecidas aos animais $(\mathrm{P}>0,05)$. As vacas que receberam ração constante durante todo período de transição produziram em média de $29,0 \pm 8,2$ litros de leite/dia, enquanto as que receberam tratamento tradicional, ração com maior teor de concentrado após o parto, produziram em média 29,1 \pm 7,5 litros de leite/dia. Os resultados obtidos não permitiram obter resultados conclusivos sobre uma recomendação da estratégia em atrasar o fornecimento de dietas ricas em concentrados para vacas leiteiras logo após o parto com objetivo de evitar possíveis oscilações na
\end{abstract}

\footnotetext{
1 Zootecnista, Extensionista da EMATER, Instituto Paranaense de Assistência Técnica e Extensão Rural, município de Inácio Martins, PR. E-mail: mjgscala@hotmail.com

2 Prof. Dr. do Dept ${ }^{\circ}$ de Zootecnia da Universidade Estadual de Londrina, UEL, Londrina, PR. E-mail fregonesijaf@hotmail.com

3 Prof ${ }^{\mathrm{a}}$. Dra . do Dept ${ }^{\mathrm{a}}$ de Zootecnia da UEL, Londrina, PR. Pesquisadores do CNPq. E-mail: mizubuti@uel.br

4 Profs. Drs. do Animal Welfare Program, University of British Columbia. E-mail: danweary@interchange.ubc.ca; nina@ interchange.ubc.ca

5 Pesquisador Dr., Agriculture and Agri-food Canada, BC, Canada. E-mail: veirad@agr.gc.ca

6 Doutoranda em Ciência Animal, Universidade Estadual de Londrina. Bolsista CAPES. E-mail: awandressa@yahoo.com.br

7 Assistente de pesquisa, M.e University of British Columbia. E-mail: lizanne.steunenberg@gov.bc.ca

* Autor para correspondência
}

Recebido para publicação 09/04/2010 Aprovado em 16/09/2011 
ingestão de matéria seca (IMS) no início da lactação.

Palavras-chave: Comportamento alimentar, manejo alimentar, período de transição, produção de leite, vacas holandesas

\begin{abstract}
Dairy cows experience challenges during the transition period, which may put them at risk of diseases such as rumen acidosis and laminitis. The traditional recommendations during the transition period is to feed dairy cows on moderate level of concentrates in the pre-partum and switch it to high grain diet just after calving. However, there are some evidences that this management may cause a bump effect on the feeding intake in the post-partum period. The aim of this work was to study the effect of a constant diet during all transition period on the behaviour; feeding intake and milk yield of dairy cows. Thirty primiparous and multiparous Holstein dairy cows were at random allocated in one group fed on higher fibre diet in the pre-partum followed by a higher concentrate diet in the post-partum (traditional management) and in a second group fed constantly on a higher fibre diet during all transition period. The behaviour and the feeding intake were monitored by an electronic feeding system and data loggers and milk yield data obtained in the milking parlour computer. The animals fed on the constant diet spent more time eating ration in second day post-partum, 142 minutes/day and cows in traditional recommendations 105 minutes/day, while cows fed on lactation diet just after calving had higher dry matter intake in day 1 averaging $2.2 \mathrm{~kg} /$ day $/ 100 \mathrm{~kg} \mathrm{LW}$ but not in the following days, longer for cows receiving the constant diet the DMI was $1.7 \mathrm{~kg} /$ day $/ 100 \mathrm{~kg} \mathrm{LW}$. There were no significant differences between the two diets related to milk yield $(\mathrm{P}>0.05)$. Cows fed diets constant throughout the transition period produced an average of $29.0 \pm 8.2$ liters of milk/day, while those who received traditional treatment ration with the highest concentrate after birth, produced an average of $29.1 \pm 7.5$ liters of milk/day. These results do not allow to a final conclusion about the recommendation to delay or not to switch diet after post-partum to avoid the bump in dry matter intake during early post-partum. Key words: Feeding behaviour, feeding management, transition period, milk production, Holstein cows
\end{abstract}

\section{Introdução}

Durante o período de transição (comumente definido como o período de três semanas antes até três semanas depois do parto) as vacas leiteiras estão altamente susceptíveis aos distúrbios metabólicos (GOFF; HORST, 1997). Nesse período, a vaca deixa de receber uma dieta rica em fibra e baixa em concentrado, para receber uma dieta alta em concentrado e pobre em fibra. Animais que não passam por uma adaptação a essa dieta rica em grãos são mais susceptíveis à acidose ruminal (OWENS; GOETSCH, 1988).

No manejo tradicional em rebanhos de alta produção, a bezerra é separada logo após o parto, e a vaca rapidamente se dirige ao cocho para se alimentar (GRANT; ALBRIGHT, 1995). Resultados de pesquisas recentes mostram um aumento de 28 \% na IMS (Ingestão de Matéria Seca) nas 24 horas pós-parto, Porém, volta a ocorrer uma queda de $11 \%$ da IMS no dia seguinte ao parto, em animais não adaptados (HUZZEY et al., 2007). Esse comportamento sugere que a ingestão rápida de grande quantidade de carboidratos fermentáveis, em animais não adaptados, leva ao acúmulo de ácidos orgânicos combinado com a inabilidade da vaca em tamponar esses ácidos, através de secreção de saliva, causando queda no $\mathrm{pH}$ do rúmen (OETZEL; NORLUND; GARRETT, 1999). Esses componentes associados podem levar à acidose, que é um dos fatores que contribuem para o aparecimento da pododermatite não infecciosa em bovinos leiteiros (MANSON; LEAVER, 1988; NOCEK, 1997), caracterizada por alterações na produção de queratina, hemorragia e úlcera da sola do casco (MURRAY; SINGH; WARD, 1993).

Os produtores de leite são geralmente orientados 
a maximizarem o consumo de alimento das vacas durante o pré-parto, com a finalidade de preparálas para um maior consumo de matéria seca (MS) logo após o parto, na tentativa de reduzir o déficit energético e desordens metabólicas, comuns do início da lactação (GRUMMER, 1995). Alguns pesquisadores (MINOR et al., 1998; DANN; VARGA; PUTNAM, 1999; HOLCOMB et al., 2001), têm mostrado que o aumento da densidade nutricional da dieta de vacas, durante o pré-parto, melhora o metabolismo e a produção dos animais no pós parto. Uma prática, comumente observada em fazendas leiteiras, é fornecer aos animais uma dieta com teor intermediário de concentrados nas últimas semanas pré-parto, a qual é substituída por outra com alto teor de concentrados logo após o parto. Embora esse manejo alimentar tenha como objetivo adaptar o rúmen à dieta com alto teor de concentrado, e ao mesmo tempo aumentar o consumo de MS, aparentemente, tem aumentado o risco de acidose do rúmen e conseqüentemente, de pododermatite (NOCEK, 1997). Alguns levantamentos têm mostrado alta prevalência de enfermidades podais em bovinos (ESPEJO; ENDRES; SALFER, 2006), inclusive em rebanhos leiteiros no Brasil (SOUZA et al., 2007).

Tendo em vista a importância do manejo alimentar no período de transição de vacas leiteiras, especialmente logo após o parto, este trabalho foi realizado com o objetivo de avaliar o comportamento alimentar, o consumo de alimentos e a produção em vacas leiteiras submetidas a diferentes manejos logo após o parto.

\section{Material e Métodos}

O experimento foi conduzido na Unidade de Pesquisas em Gado Leiteiro da University of British Columbia - UBC, Agassiz, BC, Canadá, no período de janeiro a abril de 2007. Durante o período experimental, 30 vacas da raça Holandesa (11 primíparas e 19 multíparas) foram alocadas em dois grupos de 15 animais: peso vivo $=674 \pm 96 \mathrm{~kg}$; ordem de parto $=2,1 \pm 1,33$ e condição corporal $=$ 3,2 $\pm 0,20$ (escore de 1 a 5, segundo EDMONSON et al., 1989). As informações relativas às medidas de Peso Vivo (PV) e Escore da Condição Corporal (ECC) foram obtidas realizando-se seis pesagens e seis medições do PV e do ECC, durante os prováveis dez dias que antecediam o parto e também a partir do oitavo dia após o parto. Para obtenção do PV, as vacas eram pesadas de preferência na mesma hora do dia, após a ordenha da manhã.

O estábulo tinha estrutura de madeira, pé direito de dez metros, ventilação natural e laterais abertas, mas com cortinas. Os animais foram agrupados em dois currais pré-parto e um curral pós-parto em confinamento com seis baias individuais (tipo Freestall), cada um. As baias eram preenchidas com colchões (Pasture Mat, Promat Inc., Woodstock, Ontário, Canadá) e sobre os mesmos eram colocados $0,10 \mathrm{~m}$ de areia limpa de rio; também possuíam seis comedouros da marca Insentec (Insentec, Marknesse, Holland) e um bebedouro da marca Insentec (Insentec, Marknesse, Holland). A densidade animal foi mantida em seis vacas por curral, em baias individuais, durante todo experimento. A composição dos grupos era dinâmica com vacas entrando e saindo do experimento dependendo das suas datas previstas de parto. As vacas entravam em um curral semelhante aos experimentais aproximadamente 21 dias antes da data prevista do parto e faltando 10 dias para o parto eram alocadas em um curral pré-parto para início do monitoramento. As vacas foram designadas aos tratamentos de acordo com o número de parições, PV e ECC.

Quando uma vaca mostrava sinais eminentes de parto (aumento do úbere, descida de leite, relaxamento dos ligamentos da cauda) era levada para um curral maternidade onde permanecia até a finalização do parto. O curral maternidade tinha os mesmos componentes que os currais experimentais, exceto que todas as divisórias (tubos galvanizados) das baias foram retiradas e a cama foi preenchida com serragem. No máximo três vacas por vez eram 
mantidas nesse curral maternidade e logo após o parto eram transferidas para o curral pós-parto onde eram monitoradas por mais sete dias. Foram colocadas três câmeras (CCTV) no curral maternidade para monitorar os partos. $\mathrm{O}$ equipamento para filmagem era composto de câmeras modelo Panasonic WVBP334, Osaka, Japão. As câmeras foram acopladas a um gravador videocassete (Panasonic AG-6540 ${ }^{\circledR}$ ) e um vídeo Multiplexer (Panasonic WJ-FS $416^{\circledR}$ ). Lâmpadas vermelhas de $100 \mathrm{~W}$, foram colocadas a $10 \mathrm{~m}$ de altura no curral para facilitar a gravação de vídeo durante o período noturno. As vacas foram identificadas no vídeo usando um único símbolo localizado no seu posterior e lados. Esses símbolos foram pintados nas vacas com tintura de cabelo, antes que entrassem no experimento. Animais que apresentaram sinais clínicos de doenças (cetose, hipocalcemia, retenção de placenta, metrites, mastites etc.) foram descartados do experimento. $\mathrm{O}$ momento do parto, para cada vaca, foi determinado pela gravação de vídeo e usado para expressar dados de alimentação e ingestão de água como dia zero, começando no momento que o bezerro nascia. A produção de leite individual, nas duas ordenhas diárias, era registrada diretamente no computador da ordenhadeira mecânica, e o total de leite produzido foi obtido através da soma da produção diária de cada vaca, durante dez dias.

Ao entrar no curral pós-parto as vacas eram designadas aos tratamentos de acordo com ECC, PV e números de parições: 1 - fornecimento de ração pré-parto (dieta constante) por sete dias após o parto; 2 - fornecimento de ração pós-parto (dieta tradicional). Vacas no pós-parto eram ordenhadas duas vezes por dia, aproximadamente $07 \mathrm{~h}$ e $00 \mathrm{~min}$ e $17 \mathrm{~h}$ e $00 \mathrm{~min}$. Os animais foram alimentados duas vezes por dia, aproximadamente $08 \mathrm{~h}$ e $00 \mathrm{~min}$ e $16 \mathrm{~h}$ e $00 \mathrm{~min}$ com ração totalmente misturada (RTM) durante todo período experimental, isto é, o fornecimento de ração era ad libitum.

Amostras da RTM eram coletadas em ambos tratamentos durante o período pré e pós-parto, às segundas, quartas e sextas-feiras, formando uma amostra composta. Posteriormente as amostras foram secas a $60 \pm 5^{\circ} \mathrm{C}$ por dois dias e agrupadas, formando amostras mensais, para determinação do conteúdo de matéria seca (MS) no laboratório da fazenda experimental. Para análise de MS, as amostras foram descongeladas à temperatura ambiente, homogeneizadas manualmente e colocadas em pratos de papel alumínio, procedendose a pré-secagem em estufa de ventilação forçada à temperatura de $55^{\circ} \mathrm{C}$, por 72 horas. Em seguida, foram moídas em moinho tipo Willey do modelo padrão 4 (Arthur H. Thomas Co., Filadélfia, PA), com peneira de $1 \mathrm{~mm}$ e homogeneizadas. As amostras secas semanalmente foram compostas em amostras mensais e enviadas para análises de nutrientes (Cumberland Valley Analytical Services Inc., Maugansville, MD) para determinar a proteína bruta (PB), fibra em detergente ácido (FDA), fibra em detergente neutro (FDN), nutrientes digestíveis totais (NDT) e conteúdo de energia liquida (EL) durante os quatro meses de experimento.

A Tabela 1 mostra os ingredientes e a composição química das rações dos tratamentos 1 e 2. Os tratamentos foram:

T1-Dieta Constante: Relação concentrado: volumoso 30:70. As vacas deste tratamento receberam esta ração, no período pré parto, e continuaram recebendo a mesma ração fornecida durante a fase do pré-parto, por um período de sete dias após

T2-Dieta Tradicional: Relação concentrado: volumoso 50:50. As vacas deste tratamento receberam, durante a fase pré-parto, ração pré-parto, e logo após o parto receberam a ração para vacas em lactação, isto é, ração pós-parto.

Um sistema eletrônico de alimentação (Insentec) de alta precisão, validado por Chapinal et al. (2007), foi usado para monitorar individual e continuamente o consumo de ração e água e o comportamento alimentar (tempo gasto comendo ração e bebendo água) de todos os animais experimentais. Cada vaca tinha um único transponder (chip) passivo (High 
Performance ISO Half Duplex Electronic ID Tag, Allflex, St. Hyacinthe, Quebec, Canadá) preso à sua orelha. Quando uma vaca se aproximava do cocho, uma antena detectava o seu transponder e a porta do cocho se abria, permitindo que esse animal tivesse acesso a RTM ou água. No momento que o portão do cocho se abria, o sistema Insentec registrava o tempo e o peso inicial do conteúdo do cocho. Quando uma vaca saia do cocho o portão era fechado automaticamente e o sistema novamente registrava o tempo e peso do conteúdo do cocho. Esses dados foram usados para registrar a duração de cada visita ao cocho e a quantidade de alimento e água consumidos. As medições do consumo de MS foram obtidas pela correção semanal dos consumos de alimento fresco para o conteúdo de MS da ração.

O tempo total despendido deitada e em estação foram mensurados para todas as vacas, durante todo período pré e pós-parto, usando data loggers (Tinytag Plus Gemini Inc. Chichester, UK). Os data loggers eram fixados nas pernas posteriores, logo abaixo dos jarretes, no dia anterior do início da coleta e registrava, a cada minuto, o comportamento de repouso e em estação. A cada sete dias, os dados eram transferidos para o computador, e os loggers fixados à perna contra-lateral.

Tabela 1. Composição química e ingredientes das rações dos tratamentos 1 (dieta constante 7 dias depois do parto) e 2 (dieta tradicional).

Percentagem (\%) dos ingredientes nas dietas

Ingredientes

Dieta Constante

Silagem de milho

Feno de alfafa

Silagem de gramíneas

Feno de gramíneas

Concentrado

Composição química

MS (\%)

PB (\% MS)

FDA (\% MS)

FDN (\% MS)

NDT $(\% \mathrm{MS})$

EL (Mcal/lb)

25

17

25

3

30

56,4

14,3

32,4

46,1

63,1

0,65
Dieta Tradicional

20

7

20

3

50
17,0

21,5

35,9

70,7

0,74
As análises estatísticas foram realizadas utilizandose o procedimento GLM do SAS (SAS INSTITUTE, 2003), sendo que cada animal foi considerado uma unidade experimental, $(\mathrm{n}=30)$, isto é, os animais usados podem ser considerados como amostras aleatórias de uma população de animais. As vacas foram distribuídas entre os tratamentos, a cada parto, de acordo com número de partos, ECC e PV; Três períodos diferentes foram designados para análise de variância (GLM), relacionado ao consumo de MS: dias do pós-parto $(+1 \mathrm{a}+2 ;+2 \mathrm{a}+3 \mathrm{e}+1 \mathrm{a}+4)$. Dia +1 foi considerado a partir do momento do parto de cada vaca já definido anteriormente. Diferenças entre os períodos foram testados usando t-test a $5 \%$ de probabilidade. 


\section{Resultados e Discussão}

Os resultados relativos a comportamento alimentar, tempo despendido comendo ração e consumo de ração e água entre o dia 1 ao dia 4 estão sumarizados na Tabela 2 .

Tabela 2. Tempo médio despendido i ngerindo ração, ingestão de matéria seca e de água das vacas Holandesas tratadas com dieta constante ou dieta tradicional durante os 4 dias pós-parto.

\section{Dias pós parto}

\begin{tabular}{lcccc}
\hline Item & dia-1 & dia-2 & dia-3 & dia-4 \\
\hline Tempo ingerindo ração (min. / dia) & & & & \\
Dieta constante & $123 \pm 10,9 \mathrm{a}$ & $142 \pm 9,3 \mathrm{a}$ & $171 \pm 11,6 \mathrm{a}$ & $165 \pm 10,6 \mathrm{a}$ \\
Dieta tradicional & $102 \pm 6,4 \mathrm{a}$ & $105 \pm 9,3 \mathrm{~b}$ & $128 \pm 7,3 \mathrm{a}$ & $126 \pm 8,3 \mathrm{a}$ \\
IMS (kg / dia / 100 kg PV) & & & \\
Dieta constante & $1,7 \pm 0,1 \mathrm{a}$ & $2,0 \pm 0,1 \mathrm{a}$ & $2,4 \pm 0,1 \mathrm{a}$ & $2,2 \pm 0,2 \mathrm{a}$ \\
Dieta tradicional & $2,2 \pm 0,1 \mathrm{~b}$ & $2,0 \pm 0,2 \mathrm{a}$ & $2,3 \pm 0,1 \mathrm{a}$ & $2,3 \pm 0,1 \mathrm{a}$ \\
Ingestão de água (kg / dia) & & & & \\
Dieta constante & $49 \pm 3,0 \mathrm{a}$ & $48 \pm 3,8 \mathrm{a}$ & $58 \pm 4,9 \mathrm{a}$ & $58 \pm 4,2 \mathrm{a}$ \\
Dieta tradicional & $58 \pm 3,3 \mathrm{a}$ & $55 \pm 3,6 \mathrm{a}$ & $60 \pm 3,7 \mathrm{a}$ & $62 \pm 3,4 \mathrm{a}$ \\
\hline
\end{tabular}

Médias seguidas de letras diferentes, na coluna, diferem entre si $(\mathrm{P}<0,05)$ pelo teste $t$.

\section{Comportamento alimentar}

Quando as vacas foram manejadas para que continuassem a receber a mesma ração (dieta constante) do pré-parto, durante os quatro dias seguintes ao parto, elas despenderam maior tempo no cocho comendo no dia dois após o parto quando comparado com vacas tratadas com dieta tradicional no pós-parto (Tabela 2). Ainda que resultados significantes não tenham sido confirmados para o restante do período, observou-se uma tendência das vacas tratadas com ração constante despenderem mais tempo nos cochos comendo (Figura 1). Esse resultado não surpreende, pois o conteúdo maior de fibras e menor de concentrados no tratamento constante provavelmente deve ter obrigado os animais a passarem mais tempo comendo na tentativa de ajustar sua IMS (DeVRIES; Von KEYSERLINGK; BEAUCHEMIN, 2003).
Uma provável limitação ao manejo de se atrasar a troca de ração pré-parto por uma pósparto é o fato do tempo gasto na ordenha limitar o tempo gasto comendo pelas vacas após o parto (HUZZEY et al., 2007). Apesar de as vacas leiteiras serem altamente consistentes quanto ao tempo despendido comendo, outros meios de comportamento podem ser usados para ajudar sua IMS diária (DeVRIES; Von KEYSERLINGK; BEAUCHEMIN, 2003).

\section{Manejo alimentar e IMS}

Observou-se no dia um do pós parto, nas vacas do grupo que receberam dieta tradicional, maior IMS $(\mathrm{P}<0,05)$, mas não foi observado diferença $(\mathrm{P}>0,05)$ do segundo ao quarto dia após o parto (Tabela 2). 
Figura 1. Tempo médio despendido, pelas vacas Holandesas comendo ração pré-parto (tratamento $1=$ dieta constante) ou com troca de ração pós-parto (tratamento $2=$ dieta tradicional) durante o período de transição de sete dias antes e após o parto.

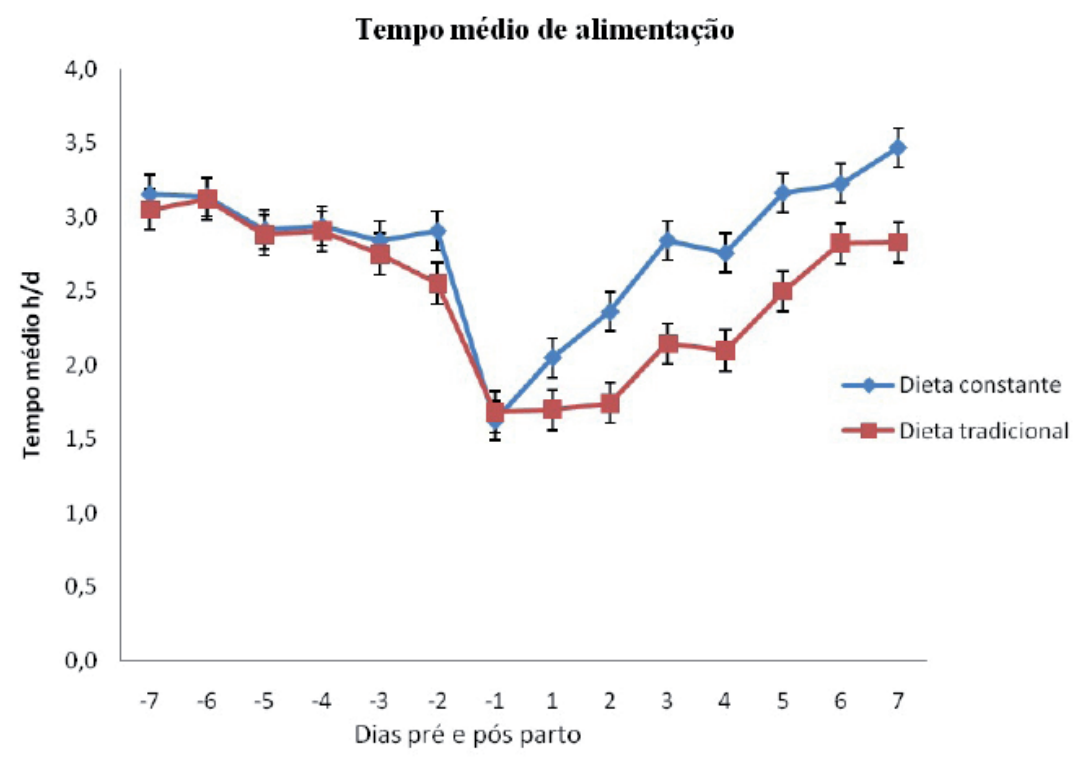

Uma das recomendações, aos produtores de leite, é maximizar a ingestão de matéria seca (IMS), afim de preparar a vaca para o maior consumo de alimentos imediatamente após o parto, com vistas a reduzir o déficit energético e, conseqüentemente, as desordens metabólicas (GRUMMER, 1995). Entretanto, essa estratégia de alimentar vacas com rações contendo alto teor de concentrados no pré-parto, não mostrou eficácia em prevenir a acidose do rúmen, quando comparada com os animais alimentados com teores moderados de concentrados (PENNER; BEAUCHEMIN; MUTSVANGWA, 2007). Há evidências de que, nas 24 horas seguintes ao parto, há um aumento de $28 \%$ na IMS, porém, esta volta a apresentar redução de $11 \%$ no dia seguinte (GRUMMER, 1995; GRUMMER; MASHEK; HAYIRLI, 2004; DANN; VARGA; PUTNAM, 1999).

Aparentemente, a mudança de dieta, de uma ração com maior teor de fibra, para uma com maior teor de concentrado, no pós parto, não afetou a ingestão de matéria seca (Figura 2), provavelmente devido ao fato dos animais que receberam a dieta com maior teor de fibra, terem compensado, com maior tempo de ingestão (Figura 1).

Durante o período de transição a vaca leiteira passa por várias situações de estresse, que podem por em risco a sua saúde (GOFF; HORST, 1997). As doenças metabólicas que afetam as vacas leiteiras, no período de transição, estão relacionadas à mudança da dieta nessa fase (CURTIS et al., 1985). Além, dessas mudanças de ambiente também enfrenta o desafio de se adaptar a uma dieta com maior teor de concentrado, e pobre em fibras em relação à que recebia anteriormente, aumentando o risco de desenvolver acidose do rúmen e pododermatite (NOCEK, 1997; STONE, 2004). 
Figura 2. Média da IMS (kg/100 kg PV/dia) de vacas Holandesas comendo ração constante (tratamento 1) ou troca de ração (tratamento 2) durante sete dias do pré e pós-parto.

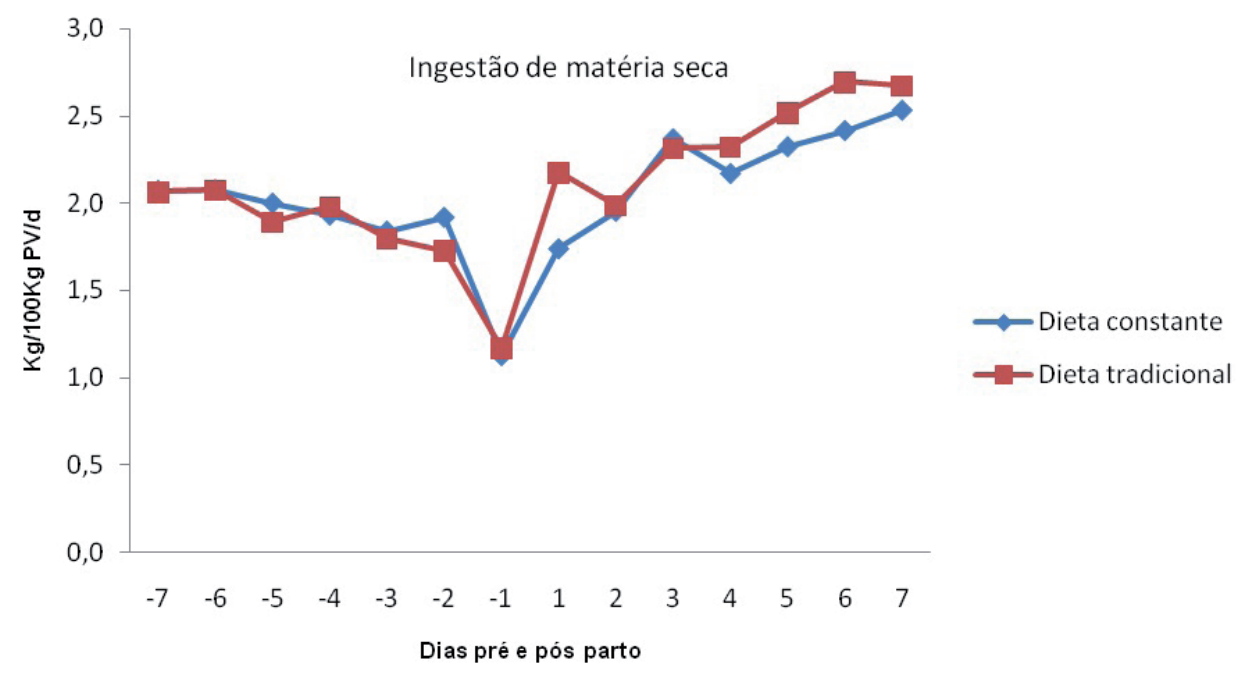

\section{Produção de leite}

Os dados relativos à produção de leite durante os 10 primeiros dias de lactação podem ser observados na Figura 3. Não foi observada diferença $(\mathrm{P}>0,05)$ na produção de leite entre as vacas holandesas que receberam a mesma dieta no período pré parto ou com maior teor de concentrado após o parto. As vacas que receberam ração constante durante todo período de transição produziram em média 29,0 \pm 8,2 litros de leite/dia, enquanto que as que receberam tratamento tradicional, ração com maior teor de concentrado após o parto, produziram em média 29,1 \pm 7,5 litros de leite/ dia.

Figura 3. Produção média de leite (litros/dia) de vacas Holandesas recebendo dieta constante durante todo período de transição ou dieta tradicional, com maior teor de concentrados por 10 dias após o parto.

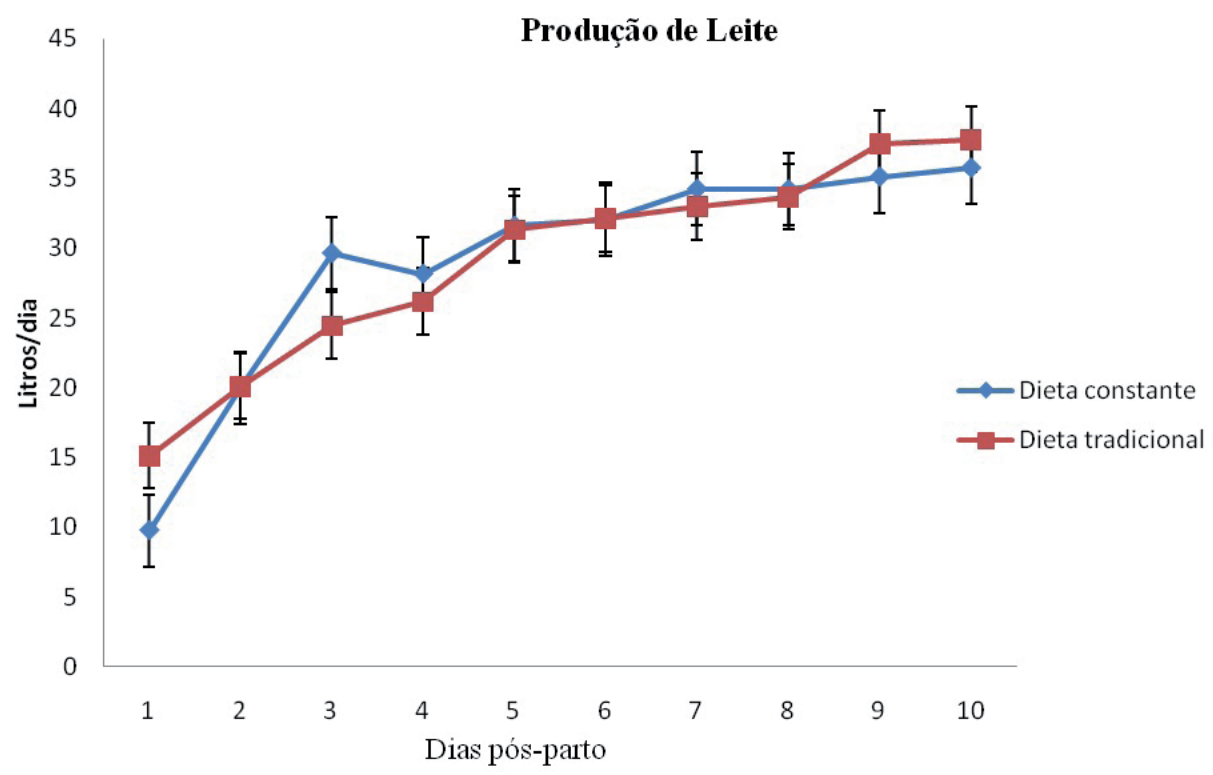


Estes resultados são contrários àqueles obtidos por Rabelo et al. (2003), que verificaram menor produção de leite quando o fornecimento da ração pós-parto era atrasada, porém naquele trabalho o fornecimento da ração com maior teor de concentrado foi atrasada por período mais longo.

\section{Medidas de comportamento}

Os resultados relativos aos comportamentos (tempo despendido em pé e deitada, número de visitas ao cocho e número de vezes que as vacas ficaram em pé), entre os 7 antes do parto (pré-parto) e 7 dias após o parto (pós-parto) estão sumarizados na Tabela 3.

Tabela 3. Média e desvio padrão de tempo despendido em pé e deitada, freqüência de visitas ao cocho e freqüência de vezes em pé para vacas Holandesas tratadas com dieta constante ou dieta tradicional, durante o período de transição de 7 dias antes e após-parto.

\section{Período}

\begin{tabular}{|c|c|c|}
\hline Item & Pré-parto & Pós-parto \\
\hline \multicolumn{3}{|c|}{ Tempo despendido em pé (horas/dia) } \\
\hline Dieta constante & $15,4+3,0 \mathrm{a}$ & $14,4+3,6 \mathrm{a}$ \\
\hline \multirow[t]{2}{*}{ Dieta tradicional } & $14,30+3 \mathrm{a}$ & $13,3+4,1 \mathrm{a}$ \\
\hline & \multicolumn{2}{|c|}{ Tempo despendido deitada (horas/dia) } \\
\hline Dieta constante & $8,60+3,0 \mathrm{a}$ & $9,6+3,6 \mathrm{a}$ \\
\hline \multirow[t]{2}{*}{ Dieta tradicional } & $9,7+2,7 \mathrm{a}$ & $10,7+4,1 \mathrm{a}$ \\
\hline & \multicolumn{2}{|c|}{ Freqüência de visitas ao cocho (n\%/dia) } \\
\hline Dieta constante & $29,7+15,0 \mathrm{a}$ & $29,8+13,9 a$ \\
\hline \multirow[t]{2}{*}{ Dieta tradicional } & $31,6+12,9 \mathrm{a}$ & $30,2+13,1 \mathrm{a}$ \\
\hline & \multicolumn{2}{|c|}{ Freqüência de vezes em pé (n\%/dia) } \\
\hline Dieta constante & $11,2+6,6 \mathrm{a}$ & $11,9+6,5 \mathrm{a}$ \\
\hline Dieta tradicional & $11,6+4,4 \mathrm{a}$ & $9,9+4,2 \mathrm{a}$ \\
\hline
\end{tabular}

Médias seguidas de letras diferentes, na coluna, diferem entre si $(\mathrm{P}<0,05)$ pelo teste $t$.

Peso vivo (PV) e escore da condição corporal $(E C C)$

A condição corporal ao parto pode afetar a IMS e produção de leite de vacas leiteiras (INGVARTSEN; ANDERSEN, 2000). Os animais utilizados neste trabalho apresentavamse em boas condições corporais durante o período experimental, o que provavelmente pode ter contribuído para manter bons níveis de consumo de ração e produção de leite.

Os resultados relativos às medidas de $\mathrm{PV}$ e ECC, coletados entre os dias $-10,-9,-8$ do período pré-parto, e os dias 8,9 e 10 do período pós-parto, estão sumarizados na Tabela 4. 
Tabela 4. Média de peso vivo $(\mathrm{kg})$ e escore da condição corporal das vacas Holandesas, tratadas com dieta constante ou dieta tradicional, durante o período de transição dos dias $-10,-9$ e -8 antes do parto e os dias 10, 9 e 8 após-parto.

\section{Período}

\begin{tabular}{lll}
\cline { 2 - 3 } Item & Pré-parto & Pós-parto
\end{tabular}

\begin{tabular}{lccc}
\hline & & Peso Vivo & \\
Dieta constante & $739,8+110,5 \mathrm{~kg} \mathrm{a}$ & & $668,9+103,9 \mathrm{Kg}$ a \\
Dieta tradicional & $723,8+92,1 \mathrm{Kg} \mathrm{a}$ & & $647,78+82,1 \mathrm{Kg} \mathrm{a}$ \\
\hline & & ECC & \\
Dieta constante & $3,5+0,1 \mathrm{a}$ & & $3,1+0,2 \mathrm{a}$ \\
Dieta tradicional & $3,2+0,2 \mathrm{a}$ & $3,0+0,2 \mathrm{a}$ \\
\hline
\end{tabular}

Médias seguidas de letras diferentes, na coluna, diferem entre si $(\mathrm{P}<0,05)$ pelo teste $t$.

\section{Conclusões}

Pode-se concluir que a mudança na relação volumoso:concentrado (30:70 para 50:50) proporcionou maior consumo de ração no primeiro dia pós-parto, mas não nos sete dias seguintes. Também foi observado redução no tempo de alimentação no segundo dia, o que não influenciou a produção de leite durante o período experimental de sete dias pós parto. Os resultados obtidos não permitem a recomendação da estratégia de atrasar o fornecimento de dietas ricas em concentrados para vacas leiteiras logo após o parto, com objetivo de evitar possíveis oscilações na ingestão de matéria seca (IMS) no início da lactação.

\section{Referências}

CHAPINAL, N.; VEIRA D. M.; WEARY, D. M.; von KEYSERLINGK, M. A. G. Technical note: Validation of a system for monitoring individual feeding and drinking behavior and intake in group-housed cattle. J. Dairy Sci., Champaign, v. 90, n. 12, p. 5732-5736, 2007.

CURTIS, C. R.; ERB, H. N.; SNIFFEN, C. H.; SMITH, R. D.; KRONFELD, D. S. Path analysis of dry period nutrition, postpartum metabolic and reproductive disorders, and mastitis in Holstein cows. J. Dairy Sci., Champaign, v. 68, n. 9, p. 2347-2360, 1985.

DANN, H. M.; VARGA, G. A.; PUTNAM, D. E. Improving energy supply to late gestation and early postpartum dairy cows. J. Dairy Sci., Champaign, v. 82, n. 8, p. $1765-1778,1999$.

DeVRIES, T. J.; Von KEYSERLINGK, M. A. G.; BEAUCHEMIN, K. A. Short communication: diurnal feeding pattern of lactating dairy cows. J. Dairy Sci., Champaign, v. 86, n. 12, p. 4079-4082, 2003.

EDMONSON, A. J.; LEAN, I. J.; WEAVER, L. D.; FARVER, T.; WEBSTER, G. A body condition scoring chart for Holstein dairy cows. J. Dairy Sci., Champaign, v. 72, n. 1, p. $68-78,1989$.

ESPEJO, L. A.; ENDRES, M. I.; SALFER, J. A. Prevalence of lameness in high-producing holstein cows housed in freestall barns in Minnesota. J. Dairy Sci., Champaign, v. 89, n. 8, p. 3052-3058, 2006.

GOFF, J. P.; HORST, R. L. Physiological changes at parturition and their relationship to metabolic disorders. J. Dairy Sci., Champaign, v. 80, n. 7, p. 1260-1268, 1997.

GRANT, R. J.; ALBRIGHT, J. L. Feeding behavior and management factors during the transition period in dairy cattle. J. Anim. Sci., Madison, v. 73, n. 9, p. 2791-2803, 1995.

GRUMMER, R. R. Impact of changes in organic nutrient metabolism on feeding the transition dairy cow. $J$ Anim. Sci., Madison, v. 73, n. 9, p. 2820-2833, 1995.

GRUMMER, R. R.; MASHEK, D. G.; HAYIRLI, A. Dry matter intake and energy balance in the transition period. Vet. Clin. North Am. Food Anim. Pract., v. 20, n. 3, p. 447-470, 2004.

HOLCOMB, C. S.; Van HORN, H. H.; HEAD, H. H.; HALL, M. B.; WILCOX, C. J. Effects of prepartum dry matter intake and forage percentage on postpartum 
performance of lactating dairy cows. J. Dairy Sci., Champaign, v. 84, n. 9, p. 2051-2058, 2001.

HUZZEY, J. M.; VEIRA, D. M.; WEARY, D. M.; Von KEYSERLINGK, M. A. G. Prepartum behavior and dry matter intake identify dairy cows at risk for metritis. $J$. Dairy Sci., Champaign, v. 90, n. 7, p. 3220-3233, 2007.

INGVARTSEN, K. L.; ANDERSEN, J. B. Integration of metabolism and intake regulation: A review focusing on periparturient animals. J. Dairy Sci., Champaign, v. 83, n. 7, p. 1573-1597, 2000.

MANSON, F. J.; LEAVER, J. D. The influence of concentrate amount on locomotion and clinical lameness in dairy cattle. Anim. Prod., Massachusetts, v. 47, n. 2, p. 185-190, 1988.

MINOR, D. J.; TROWER, S. L.; STRANG, B. D.; SHAVER, R. D.; GRUMMER, R. R. Effects of nonfiber carbohydrate and niacin on periparturient metabolic status of lactation dairy cows. J. Dairy Sci., Champaign, v. 81, n. 1, p. 189-200, 1998.

MURRAY, R. D.; SINGH, S. S.; WARD, W. R. Pathophysiology of lameness in dairy cattle. Cattle Pract., United Kingdom, v. 1, p. 322-331, 1993.

NOCEK, J. E. Bovine acidosis: implications on laminitis. J. Dairy Sci., Champaign, v. 80, n. 5, p. 1005-1028, 1997.

OETZEL, G. R.; NORLUND, K. V.; GARRETT, E. F. Effect of ruminal $\mathrm{pH}$ and stage of lactation on ruminal lactate concentrations in dairy cows. J. Dairy Sci., Champaign, v. 82, p. 38, 1999. Suplemento 1.

OWENS, F. N.; GOETSCH, A. L. Ruminal fermentation. In: $\mathrm{CHURCH}, \mathrm{D}$. C. The ruminant animal. Digestive, physiology and nutrition. Long Grove: Waveland Press, 1988. p. 145-171.

PENNER, G. B.; BEAUCHEMIN, K. A.; MUTSVANGWA, T. Severity of ruminal acidosis in primiparous holstein cows during the periparturient period. J. Dairy Sci., Champaign, v. 90, n. 1, p. 365-375, 2007.

RABELO, E.; REZENDE, R. L.; BERTICS, S. J.; GRUMMER, R. R. Effects of transition diets varying in dietary energy density on lactation performance and ruminal parameters of dairy cows. J. Dairy Sci., Champaign, v. 86, n. 3, p. 916-925, 2003.

SAS INSTITUTE. SAS user's guide. Version 9.1. SAS Institute Inc., Cary, NC. 2003.

SOUZA, R. C.; CARVALHO, A. U.; FERREIRA, P. M.; FACURY FILHO, E. J.; FERREIRA, M. G., FERREIRA, R. G.; COSTA, C. O.; MOURTHE NETO, A. Prevalence and distribution of digital lesions in dairy cows in the regions of Belo Horizonte and Pedro Leopoldo. Ciência
Anim. Bras., Goiânia, v. 8, n. 4, p. 823-831, out./dez. 2007.

STONE, W. C. Nutritional approaches to minimize subacute ruminal acidosis and laminitis in dairy cattle. $J$. Dairy Sci., Champaign, v. 87, p. 13-26, 2004. Suplemento. 
